\title{
Pulmonary Thromboembolism in a Patient with Behcet's Disease: A Case Report
}

\section{Zhang $\mathrm{W}^{1}$, Li $\mathrm{Y}^{2}$, Zhuang $\mathrm{G}^{2}$ and $\mathrm{Me}^{2 *}$}

${ }^{1}$ Department of Pediatric Cardiology, Gansu Provincial Maternity and Child-care Hospital, China

${ }^{2}$ Department of Nuclear medicine, Gansu provincial hospital, China

*Corresponding author: Kun Me, Department of Pediatric Cardiology, Gansu Provincial Maternity and Child-care Hospital, Gansu province, No.204 West Street, Donggang district, Lanzhou, China, Email: calypso122@163.com

\section{Case Report \\ Volume 6 Issue 1}

Received Date: January 13, 2021

Published Date: February 04, 2021

DOI: $10.23880 /$ oajg-16000209

\section{Abstract}

Behcet's disease is a multisystem vasculitis characterized by recurrent oral ulcers and any of several systemic manifestations. We report on a case of a 53-year-old woman with Behcet's disease who was admitted for cough and erythema nodosum. The patient had dyspnea on the third day of admission. The computed tomographic pulmonary angiography (CTPA) and SPECT pulmonary perfusion imaging showed pulmonary embolism. Pulmonary embolism is a rare complication of Behcet's disease, early diagnosis and treatments are essential for the management of Behcet's disease. Written consent for publication was obtained from the patient.

Keywords: Behcet's Disease; Pulmonary Embolism; SPECT Pulmonary Perfusion Imaging; Computed Tomographic Pulmonary Angiography

Abbreviations: CTPA:ComputedTomographicPulmonary Angiography; PTE: Pulmonary Thromboembolism; CNS: Central Nervous System.

\section{Case Description}

A 53-year-old woman with intermittent self-healing genital ulcers for 5 years was admitted to Gansu Provincial People's Hospital. The patient had lower extremities erythema nodosum, oral aphthous ulcers, and cough up small amount of white sputum for 1 month. There are different size erythema nodosums on her legs with obviously pressing pain. On the third day of hospitalization, the patient had expiratory dyspnea. The patient had hypoxemic (oxygen saturation $64 \mathrm{mmHg}$ on room air), and the remainder of the physical examination was unremarkable. She had the history of recurrent oral aphthous ulcers, dry mouth, fatigue, right shoulder pain, and both thumb interphalangeal joints pain.
Laboratory results included a creative protein concentration of $6.1 \mathrm{mg} / \mathrm{l}$, an erythrocyte sedimentation rate of $34 \mathrm{~mm} / \mathrm{h}$, positive of antinuclear antibodies nucleolus type (1:3200) and Ro-52 antibodies. The causative organisms were not identified in microbiologic studies. Computed tomography of the chest showed that pulmonary fibrosis and multiple high densities nodular scattered in bilateral lung fields and atelectasis in middle lobe of right lung (Figure 1). SPECT pulmonary perfusion imaging demonstrated the posterior segments of the upper lobe of the right lung pulmonary perfusion defects (Figure 2). CTPA showed the branch of the apical artery of the right lung occluded and stenosis at the beginning segment of the Posterior ascending artery of the right lung, thrombosis at distal of the Posterior ascending artery of the right lung (Figure 3). After analyzing both clinical and laboratory data, Behcet's disease and pulmonary thromboembolism were diagnosed. 


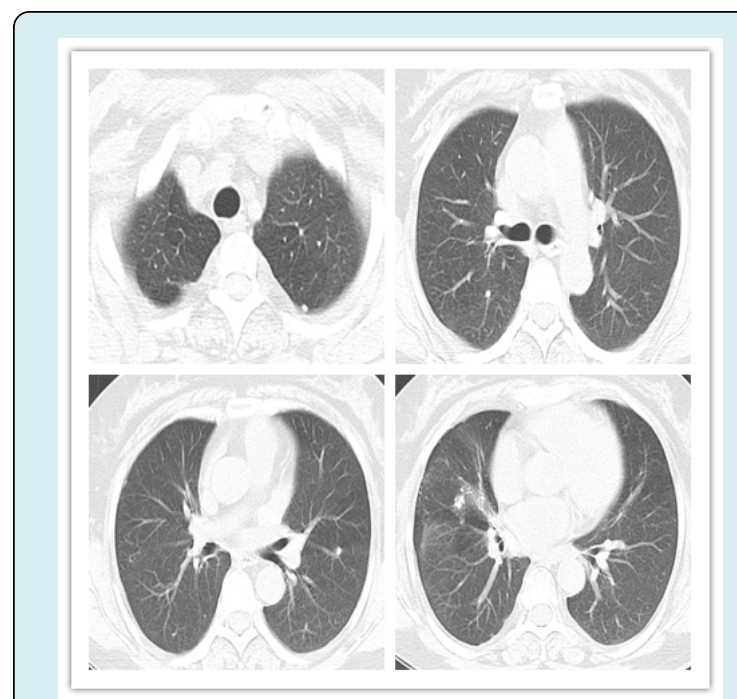

Figure 1: Computed tomography of the chest.

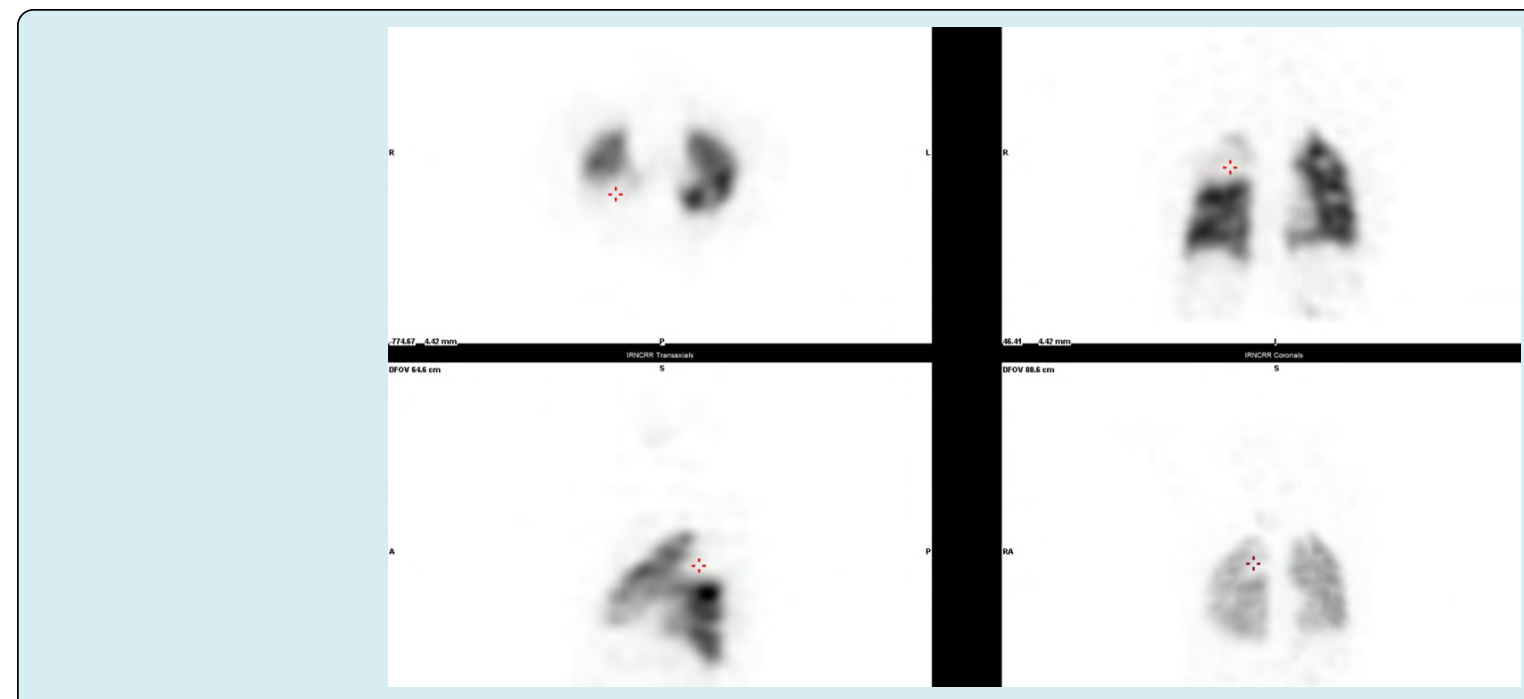

Figure 2: SPECT pulmonary perfusion imaging: The posterior segments of the upper lobe of the right lung pulmonary perfusion defects.
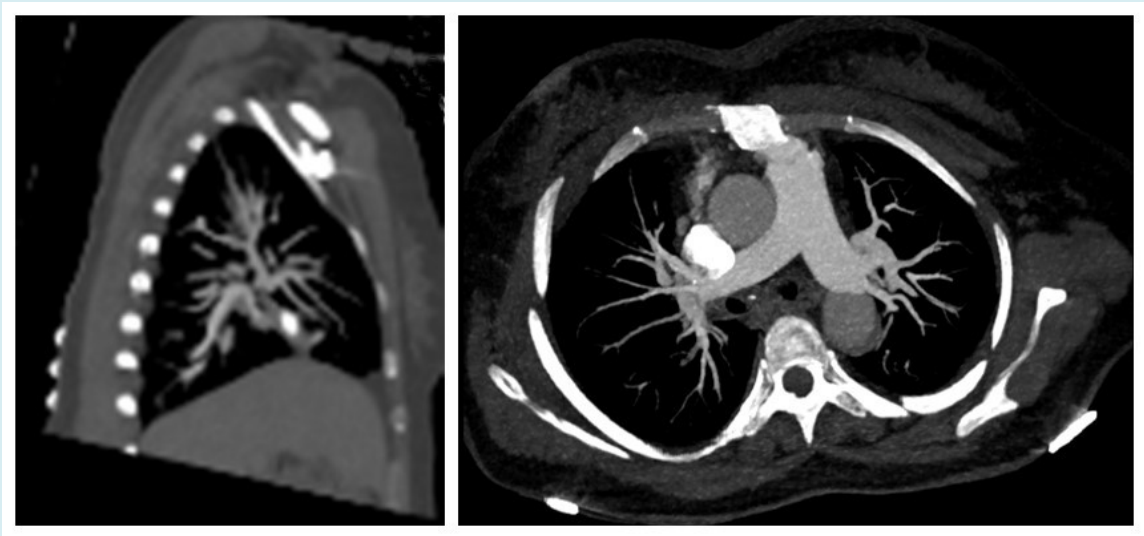

Figure 3: Computed tomographic pulmonary angiography. 


\section{Open Access Journal of Gynecology}

\section{Discussion}

Bechet's disease is a systemic and chronic inflammatory disorder that primarily known with recurrent oral ulcers and any of several systemic manifestations, including genital ulcers, ocular disease, skin lesions, arthritis, and gastrointestinal, neurologic, or vascular disease [1,2]. The main histological feature is a unique vasculitis that is not confined to a specific vessel size [3]. After reviewing of the patient's past medical history and completing screening to rule out other possible infections, clinical manifestations of the patient were decisively supported the diagnosis of BD. BD may cause more serious complications that may lead to severe morbidity and even mortality, including blindness and visual impairment, thrombotic complications such as pulmonary thromboembolism (PTE), arterial aneurysms, and central nervous system (CNS) involvement [4]. PTE can complicate BD, but there are rare reports $[5,6]$. Desbois AC, et al. [7] had reported the prevalence of pulmonary embolism linked to BD was $2 \%-27 \%$. Wu X, et al. [8] had reported the prevalence of PTE in Chinese patients with BD was 15.1 $\%$. Combined clinical manifestation with CTPA and SPECT pulmonary perfusion imaging, PTE could be diagnosed decisively. The etiologies of BD complicated with pulmonary embolism includes deep vein thrombosis, in situ thrombosis, or intracardiac thrombi $[7,9]$. The patient performed no lower limb deep vein thrombosis and echocardiography did not reveal intracardiac thrombi. Thus, we hypothesized that the etiology of pulmonary embolism was in situ thrombosis [10].

\section{Conclusion}

In summary, patients with BD may develop PTE (pulmonary embolism) and it's essential to early diagnose by combination of history of disease, physical examination, and appropriate imaging.

\section{References}

1. Mason RM, Barnes CG (1969) Behcet's syndrome with arthritis. Ann Rheum Dis 28(2): 95-103.

2. Weili Xing, Girider Swaminathan, Dorai Raj Appadorai, Ashish Anil Sule (2013) A Rare Case of Behçet Disease Presenting with Pyrexia of Unknown Origin, Pulmonary Embolism, and Right Ventricular Thrombus. International Journal of Angiology 22(3): 193-198.

3. Melikoglu M, Kural-Seyahi E, Tascilar K, Yazici H (2008) The unique features of vasculitis in Behcet's syndrome. Clin Rev Allergy Immunol 35(1-2): 40-46.

4. Davatchi F, Shahram F, Chams-Davatchi C, Shams H, Nadji A, et al. (2010) Behcet's disease: from east to west. Clin Rheumatol 29(8): 823-833.

5. Hammami R, Abid L, Frikha F, Marzouk S, Tounsi A, et al. (2012) Intracardiac thrombus in a young man: don't forget Behçet's disease!. Intern Med 51(14): 1865-1867.

6. Marc K, Iraqui G, Jniene A, Benamor J, Bourkadi JE (2008) Intracardiac thrombus and pulmonary artery aneurysm in Behcet's disease. Rev Mal Respir 25(1): 69-72.

7. Desbois AC, Wechsler B, Cluzel P, Helft G, Boutin D, et al. (2014) Cardiovascular involvement in Behçet's disease. Rev Med Interne 35(2): 103-111.

8. Wu X, Li G, Huang X, Wang L, Liu W, et al. (2014) Behçet's disease complicated with thrombosis : a report of 93 Chinese cases. Medicine(Baltimore) 93(28): e263.

9. Calamia KT, Schirmer M, Melikoglu M (2011) Major vessel involvement in Behçet's disease: an update. Curr Opin Rheumatol 23(1): 24-31.

10. Kobayashi T, Kishimoto M, Swearingen CJ, Filopoulos MT, Ohara Y, et al. (2013) Differences in clinical manifestations, treatment, and concordance rates with two major sets of criteria for Behçet's syndrome for patients in the US and Japan: data from a large, threecenter cohort study. Mod Rheumatol 23(3): 547-553. 